\title{
Programa D-Valor: evaluación del servicio de dispensación de bifosfonatos
}

\author{
Luis Salar Ibáñez ${ }^{1}$ Nancy Solá2, Raquel Cámara ${ }^{3}$, Ana Cosín ${ }^{3}$, Ana Dago ${ }^{4}$, Pedro Gutiérrez ${ }^{3}$ \\ 1. Farmacéutico comunitario. Valencia. 2. Profesora asociada. Departamento de Fisiología, Farmacología y Toxicología. UCH-CEU. Moncada (Valencia).
} 3. Farmacéutico comunitario. Madrid. 4. Directora revista Pharmaceutical Care España.

\section{PALABRAS CLAVE}

farmacia comunitaria, servicio de dispensación, bifosfonatos

\section{KEYWORDS}

community pharmacy, dispensing service, bisphosphonates

\section{RESUMEN}

Introducción: Para que un medicamento sea efectivo y seguro es imprescindible que el paciente tenga unos conocimientos adecuados sobre el uso de ese medicamento: indicación, posología, forma de uso y duración del tratamiento, entre otros. Además, en el caso de los bifosfonatos también debe asegurar unos aportes adecuados de calcio.

Objetivos: Evaluar el conocimiento del paciente, uso de calcio y percepción de efectividad y seguridad del bifosfonato.

Material y método: Estudio observacional prospectivo multicéntrico realizado en farmacias comunitarias de España. Entrevista estructurada ante la demanda con receta de cualquier bifosfonato. Se registraron los conocimientos del paciente sobre los cuatro conceptos citados, si es tratamiento de inicio o de continuación, su percepción de efectividad y seguridad, la existencia de aporte suficiente de calcio y la actuación e intervención final del farmacéutico.

Resultados: 2.527 farmacéuticos de toda España registraron 199.957 dispensaciones, de ellas 37.083 fueron bifosfonatos. Los pacientes fueron $93 \%$ mujeres, mayoritariamente mayores de 65 años. El $7 \%$ no conocía indicación, posología 4\%, forma de uso $11 \%$ y duración del tratamiento $49 \%$. Si el tratamiento es de inicio hubo más carencias de información. La percepción de efectividad y seguridad siempre fue menor si el paciente desconocía alguno de los conceptos citados. Un 12\% no aseguraba el aporte diario de calcio entre dieta y suplementos.

Discusión: Un porcentaje importante de pacientes tiene carencias de información que dificultarán la efectividad y/o seguridad de su tratamiento con bifosfonatos. El farmacéutico está en una posición magnífica para detectar y corregir estas carencias, mejorando el conocimiento del proceso de uso de estos medicamentos.

\section{D-Valor program: evaluation of the bisphosphonate dispensation service}

\section{ABSTRACT}

Introduction: In order that a drug may be effective and safe, the patient must have a proper knowledge of the use of it: indication, dosage, method of use and treatment duration, inter alia. Furthermore, in the case of bisphosphonates calcium supplements must also be ensured.

Objetives: Evaluate the knowledge of the patient, their use of calcium and their perception of the effectiveness and safety of bisphosphonates.

Material and method: Prospective observational study implemented in Spanish community pharmacies. Structured interview when a request with prescription of any bisphosphonate is made. The following pieces of information are recorded: the knowledge of the patient about the 4 aforementioned items, whether it is initial therapy or continuation treatment, the perception by the patient of the effectiveness and safety of the drug, the existence of sufficient input of calcium and the action and final intervention of the pharmacist.

Results: 2.527 pharmacists across Spain registered 199.957 dispensations, of which 37.083 were bisphosphonates. Patients were $93 \%$ female, mostly over 65 years. $7 \%$ of the patients did not know the indication, $4 \%$ the dosage, $11 \%$ the use form and $49 \%$ the duration of treatment. In the case of initial therapy the lack of information was higher. The perception of the effectiveness and safety of the drug was lower if the patient did not know any of these items. For $12 \%$ of the patients an adequate calcium input was not ensured.

Discussion: A significant proportion of patients have information gaps that hinder the effectiveness and/or safety of the treatment with bisphosphonates. The pharmacist is in an optimal position to detect and correct these deficiencies, by improving the use process of these drugs.

El programa D-Valor: El valor de la dispensación fue organizado y dirigido por las asociaciones profesionales Sociedad Española de Farmacia Comunitaria (SEFAC) y la Fundación Pharmaceutical Care España, y por el medio de comunicación profesional Correo Farmacéutico. Contó con el aval del Ministerio de Sanidad, Servicios Sociales e Igualdad y del Consejo General de Colegios Oficiales de Farmacéuticos.

El trabajo completo, del cual en este artículo se describe la parte correspondiente a bifosfonatos, fue presentado en el V Congreso Nacional de Farmacéuticos Comunitarios. 15-17/11/2012. Barcelona y en la VIII Conferencia de la PCNE (Pharmaceutical Care Network Europe) 6-8/2/2013 en Berlín. Obtuvo el premio en la categoría institucional farmacéutica en los "V Premios Esteve. Unidos por la Atención al Paciente".

Financiación: El proyecto fue financiado por Laboratorios Mylan

Conflicto de intereses: Los autores declaran no existir conflicto de intereses en relación con el contenido del presente artículo.

Cite este artículo como: Salar L, Solá N, Cámara R, Cosin A, Dago A, Gutiérrez P. Programa D-Valor: evaluación del servicio de dispensación de bifosfonatos. Farmacéuticos Comunitarios. 2014 Sep 01; 6(3):5-11. doi:10.5672/FC.2173-9218.(2014/Vol6).003.02

Autor para correspondencia: Luis Salar lbáñez (I.salar.000@micof.es).

ISSN 1885-8619 OSEFAC (Sociedad Española de Farmacia Comunitaria). Todos los derechos reservados. 


\section{Introducción}

En el proceso normal de atención sanitaria, cuando un paciente es atendido por un médico es frecuente que se le prescriba un medicamento. Si es así, el paciente acudirá a una farmacia donde se le dispensará el medicamento y después se irá a su casa donde empezará a utilizar el medicamento con la esperanza de obtener resultados positivos. Para ello es imprescindible que el paciente lo utilice correctamente, pero esta parte del proceso es la única que normalmente no está supervisada por un profesional sanitario.

Para la utilización correcta de un medicamento es imprescindible tener unos conocimientos mínimos sobre ese medicamento. Se ha considerado que lo más básico es la indicación, posología, forma de uso y duración del tratamiento (1). Aunque parece lógico que el médico informe al paciente cuando le prescribe un medicamento, no siempre es así. Y aunque el médico informe, el paciente muchas veces no lo entiende y sale de la consulta sin haber preguntado todo lo que querría (2), demandando en la farmacia mayor información (3).

Uno de los principales problemas de la farmacoterapia es que los pacientes no se adhieren a los tratamientos. La falta de información no es su única causa, pero parece ser de las más importantes (4). Muchos pacientes considerados incumplidores lo son porque no han entendido las instrucciones del médico $(5,6)$, especialmente los pacientes polimedicados con pautas complejas (7). Podemos afirmar que un enfermo más informado es un enfermo más cumplidor (8).

En el caso de los bifosfonatos estos tienen unas características especiales. Se utilizan para una enfermedad que "no duele". Hay que explicarle bien al paciente lo que puede esperar de ellos, porque no podrá valorar su efectividad. La posología es fácil y peculiar. Es fácil recordar "una pastilla a la semana” o al mes. Pero la técnica de administración es compleja. "Tragar en ayunas, sin masticar, con abundante agua y permanecer erguido durante la próxima hora" (9). La duración del tratamiento presenta menos problemas ya que se puede considerar largo. Los consensos están ahora aconsejando una duración media de 5 años y evaluar la conveniencia de continuar teniendo en cuenta el riesgo residual de fractura en ese momento (10). El caso es que la tasa de adherencia es baja, $47 \%$ en el primer año para los preparados mensuales y 30\% para los semanales (10).

La técnica de administración en los bifosfonatos es importante. Hay que asegurar un paso rápido por el esófago minimizando el contacto del medicamento con las paredes del esófago (11), porque puede producir esofagitis $(12,13)$. Por la misma razón hay que evitar el reflujo permaneciendo erguido. Incluso podría producir cáncer de esófago, aunque eso no está claro (14). Debe tomarse con estómago vacío porque su biodisponibilidad oral es muy baja, y pasa a ser despreciable si se toma junto con cualquier alimento o bebida a excepción del agua (9).

Estos cuatro conceptos asociados al adecuado proceso de uso de los medicamentos a los que nos estamos refiriendo, indicación, posología, forma de uso y duración del tratamiento, los podemos agrupar en dos: los que son imprescindibles al iniciar el tratamiento, que serian posología y forma de uso, porque si no se conocen difícilmente se puede hacer un uso correcto, y los que no son tan importantes al empezar, pero sí durante el tratamiento, la indicación y la duración del mismo. Conocer la indicación puede no ser imprescindible al iniciar el tratamiento, pero difícilmente se cumplirá mucho tiempo si no se conoce.

Además, en todas las fichas técnicas se indica que hay que asegurar la calcemia, y uno de los criterios STOP/ STARTT indica la necesidad de administrar calcio y vitamina D cuando hay osteoporosis. Se han querido relacionar los suplementos de calcio con problemas de litiasis renal y cardiacos, pero los estudios demuestran que, hoy por hoy, no hay evidencia $(15,16)$.

Por tanto, para facilitar el correcto uso de los bifosfonatos, el farmacéutico deberá asegurarse durante la dispensación que el paciente conoce la indicación, la posología, la forma de utilización y la duración del tratamiento. Además intentará verificar que el paciente toma suficiente calcio, ya sea con la dieta o con suplementos. Y dado que la forma de administración es algo peculiar y muy importante para asegurar la efectividad y la seguridad del tratamiento debe hacer especial hincapié en cómo se lo toma el paciente.

\section{Objetivos}

1. Realizar un registro de las dispensaciones de bifosfonatos realizadas en farmacias comunitarias españolas.

2. Evaluar el conocimiento del paciente acerca de la indicación, posología, forma de administración y duración del tratamiento con bifosfonatos al inicio de tratamiento y en tratamientos de continuación.

3. Evaluar el consumo de calcio e identificar pacientes con sospecha de insuficiente calcemia.

4. Evaluar la percepción del paciente sobre la efectividad y seguridad de los bifosfonatos.

5. Realizar las intervenciones farmacéuticas necesarias para resolver carencias de información u otros problemas identificados en el contexto de la dispensación de bifosfonatos.

\section{Material y métodos}

Durante los meses de enero a junio de 2012 se realizó en España el programa D-Valor, promovido por SEFAC y la Fundación Pharmaceutical Care España. En él se registraron datos de la dispensación de medicamentos pertenecientes a cinco grupos terapéuticos: antiasmáticos, benzodiazepinas, estatinas, aintiinflamatorios no esteroides y bifosfonatos. En este trabajo vamos a estudiar únicamente los bifosfonatos. Es un estudio observacional prospectivo multicéntrico con un componente de intervención.

Ante la demanda de cualquier medicamento de estos grupos, realizada por el propio paciente o su cuidador, el farmacéutico debía averiguar mediante las preguntas adecuadas si el paciente conocía la indicación, posología, forma de uso y duración del tratamiento. En el caso concreto de los bifosfonatos se insistía en la forma de uso, por ser esta especialmente importante, y además se comprobaba si tomaba suficiente calcio, Se le preguntaba si tomaba algún suplemento de calcio, y si no era así se le preguntaba por la ingesta de calcio con la dieta, especialmente con alimentos lácteos. Todo esto se hacía mediante preguntas breves para mantener la necesaria agilidad que requiere el servicio de dispensación.

También se preguntaba si era la primera vez que tomaba ese medicamento (tratamiento de inicio) o si ya 
nos lo pedía para continuar con un tratamiento iniciado anteriormente.

En el caso de que se tratara de la continuación de un tratamiento se le preguntaba por su percepción de efectividad y seguridad del medicamento. Aquí simplemente se registraba la opinión del paciente.

$\mathrm{Si}$ el farmacéutico encontraba algún problema o carencia de información debía resolverla. Se registraba también la intervención del farmacéutico, que podía ser ofrecer información personalizada del medicamento, derivar al servicio de seguimiento farmacoterapéutico, derivar al médico $u$ otro. Las diversas intervenciones no eran excluyentes. Dado que no se registraba ningún dato identificativo no podia haber ningún tipo de seguimiento. Esto impidió que se pudiera valorar el resultado de nuestra intervención.

Posteriormente el farmacéutico debía registrar todos estos datos en una web habilitada especialmente para ello. En esa web también se registraba la edad y el sexo del paciente y el código nacional del bifosfonato dispensado. También se habilitó una hoja de registro en papel para quien prefiriera hacerlo así, aunque era obligatorio registrarlo después en la web (figura 1).

Se realizó un curso de formación online, con siete temas, que todos los participantes debían superar. Cinco de los temas se correspondían con los cinco grupos de medicamentos y se

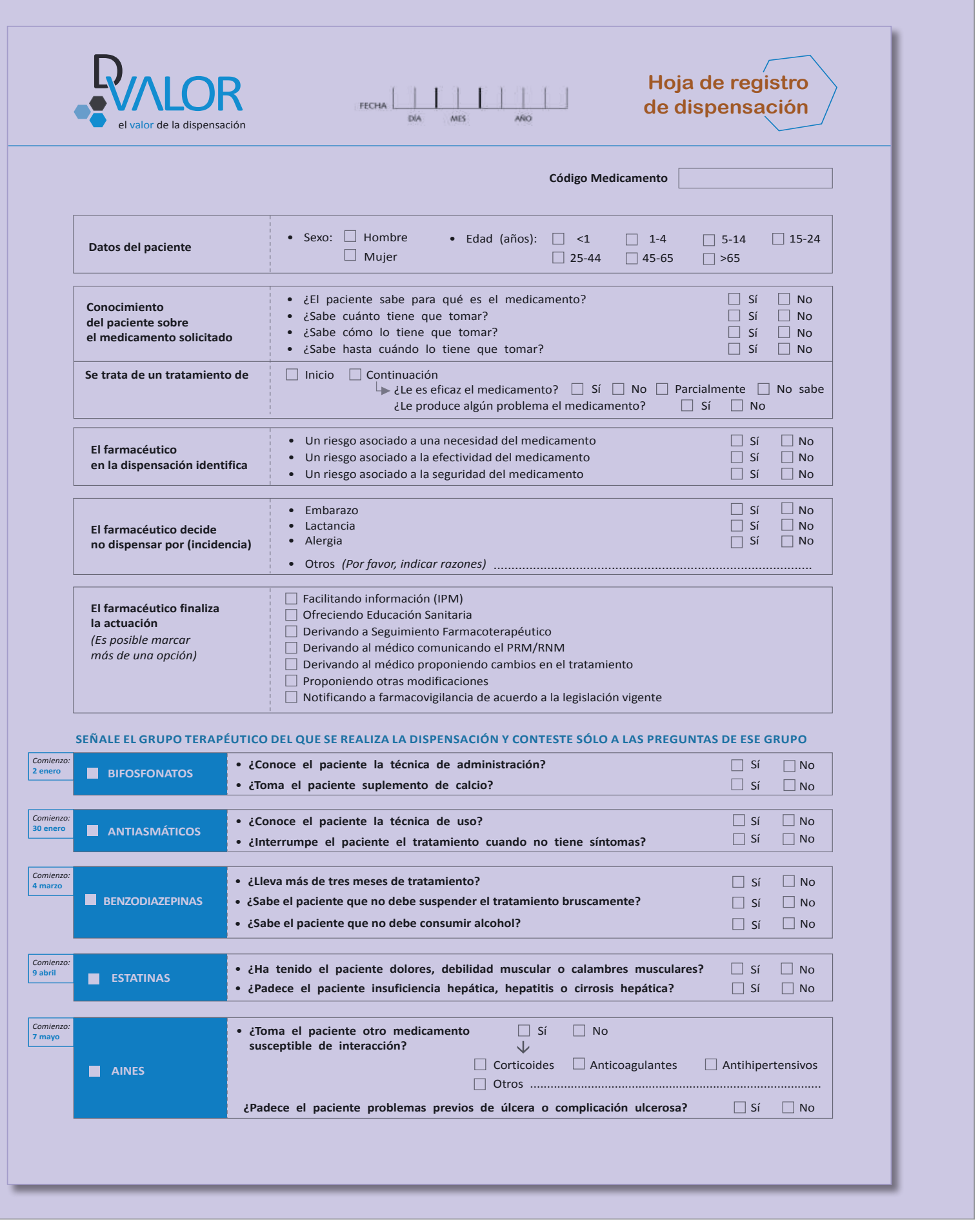

Figura 1 Hoja de registro de datos del programa D-Valor 


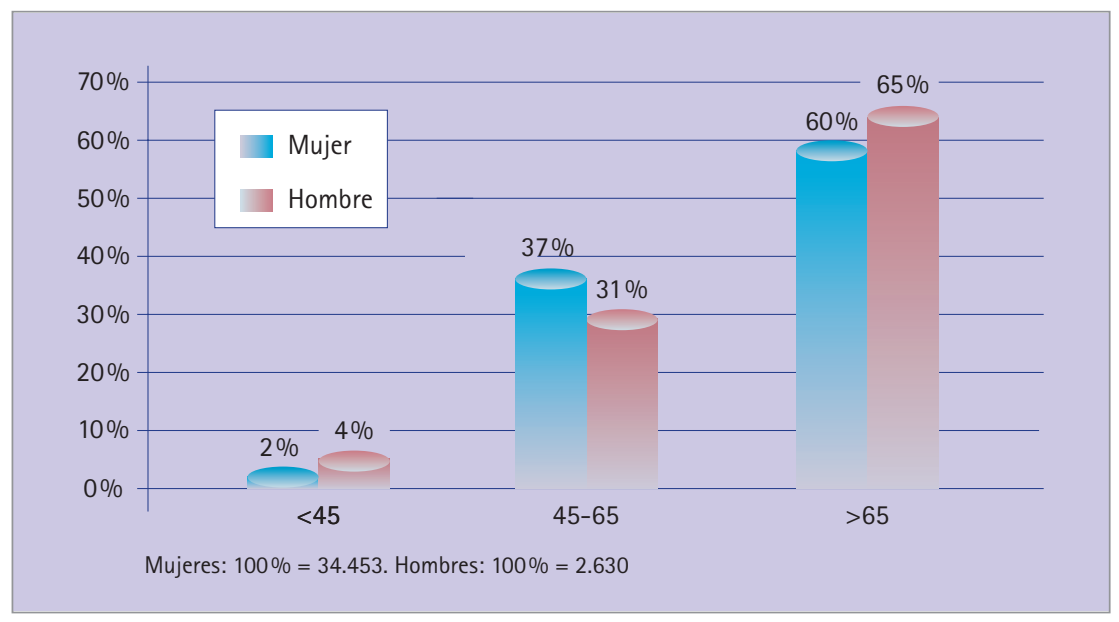

Figura 2 Distribución de los pacientes por edades en cada sexo

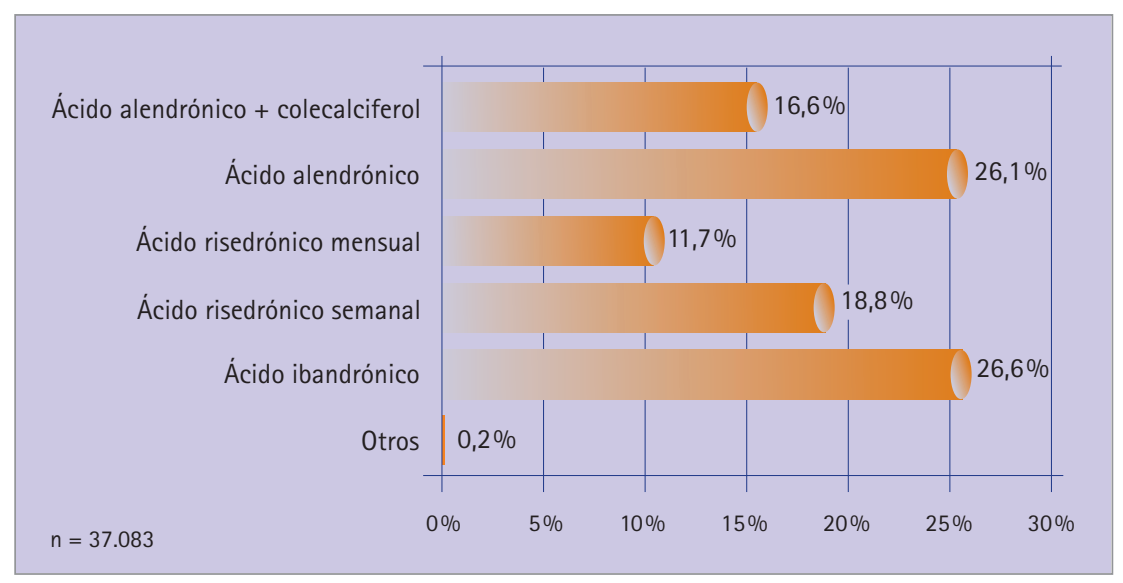

Figura 3 Distribución de las dispensaciones de bifosfonatos por principio activo

iban publicando uno al mes, al mismo tiempo que se abría el periodo de registro de ese grupo de medicamentos. Los datos se registraron en una base de datos Access ${ }^{\circledast}$ creada para el proyecto y se procesaron con Excel ${ }^{\circledast}$. Se aplicó la prueba de la $\chi^{2}$ de Pearson a las variables de conocimiento, percepción de efectividad y seguridad, suplementos de calcio e intervención y se consideró significativa una $p<0,05$.
No se consideró necesario pasar por ningún comité de ética dado que el farmacéutico lo único que hacía era registrar su intervención habitual. Por ello mismo tampoco se consideró necesario pedir al paciente que firmara ningún consentimiento y no fue necesario garantizar confidencialidad alguna ya que en ningún momento se registró ningún dato que permitiera identificar al paciente.

\section{Resultados}

En el programa D-Valor participaron 2.527 farmacéuticos que recogieron 199.957 dispensaciones. De ellas 37.083 fueron de bifosfonatos. Los pacientes que solicitaban el bifosfonato fueron $93 \%$ mujeres y $7 \%$ hombres. El grupo de edad más frecuente fue el de mayores de 65 años. En la figura 2 se indica la distribución por edades dentro de cada sexo.

Los principios activos utilizados fueron los tres mayoritarios: los ácidos alendrónico, con o sin colecalciferol, risedrónico, en presentación de dosis semanal o de dos dosis mensuales, e ibandrónico. 59 (0,16\%) fueron los ácidos clodrónico y etidrónico, medicamentos de diagnóstico hospitalario. En la figura 3 puede verse su distribución.

Hubo 121 (0,3\%) dispensaciones de ácido alendrónico diario, 174 (0,5\%) de ácido risedrónico $5 \mathrm{mg}$ también diario, y 49 (0,1\%) de ácido risedrónico $30 \mathrm{mg}$ para uso diario.

El 87\% (32.124) de los bifosfonatos solicitados fueron para tratamiento de continuación, y 4.959, el 13\%, fueron primeras dispensaciones.

Las carencias de información del paciente según fuera tratamiento de continuación o de inicio se indican en la figura 4. 19.202 (52\%) pacientes resultaron tener alguna carencia de información, siendo 3.461 (70\%) en tratamientos de inicio y 15.741 $(49 \%)$ en tratamientos de continuación $(p<0,05)$. La carencia más frecuente fue la información sobre la duración del tratamiento. Si solo tenemos en cuenta los dos conceptos considerados imprescindibles al iniciar el tratamiento, posología y forma de uso, entonces desconocen alguno de estos dos conceptos

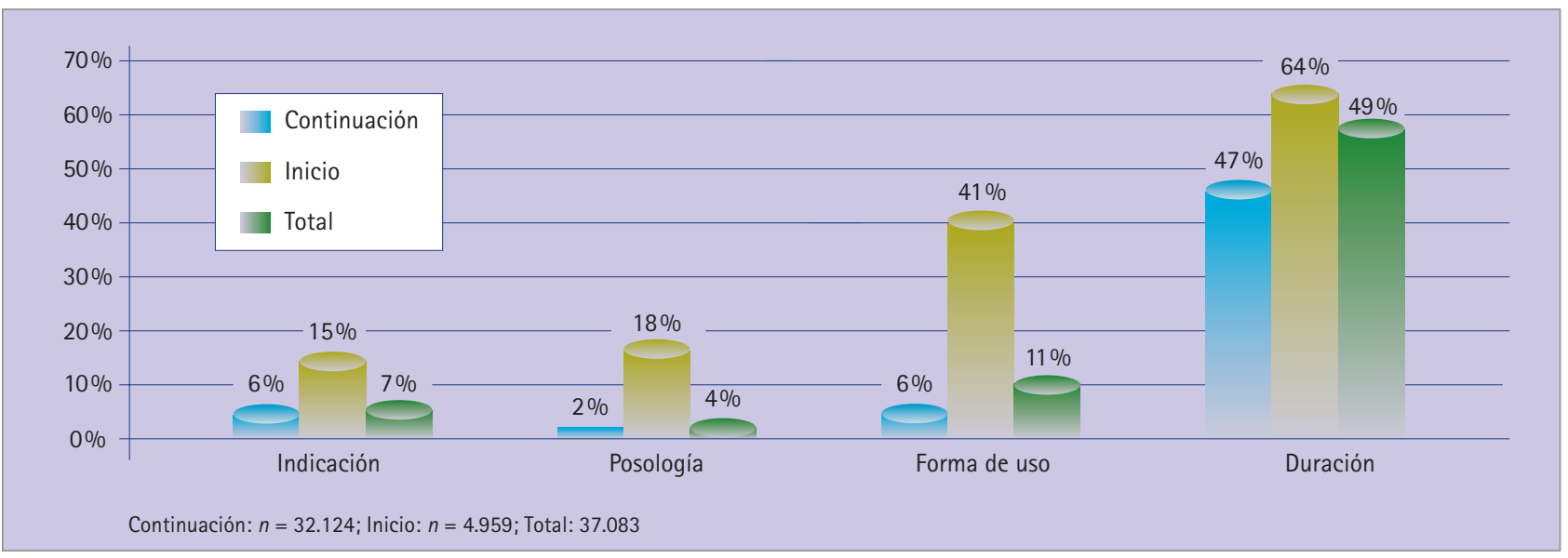

Figura 4 Carencias de información en tratamiento de inicio y continuación 
$2.127(43 \%)$ tratamientos de inicio y $2.213(7 \%)$ tratamientos de continuación $(p<0,05)$.

Los hombres tuvieron más carencia de información que las mujeres. En lo que respecta a la indicación del medicamento, el $11 \%$ de los hombres dijo no conocerlo, frente al 7\% de las mujeres $(p<0,05)$, en cuanto a la posología, el $6 \%$ frente al $4 \%(p<0,05)$ y respecto a la pauta posológica, el 15\% frente al $11 \%(p<0,05)$. Únicamente en la duración del tratamiento no hubo diferencias estadísticamente significativas.

Evaluación de la ingesta de calcio

El $12 \%$ (4.312) de los pacientes no tomaba suficiente calcio ni con la dieta ni con suplementos, de ellos el $42 \%$ estaba en tratamiento de inicio y el $7 \%$ en tratamiento de continuación.

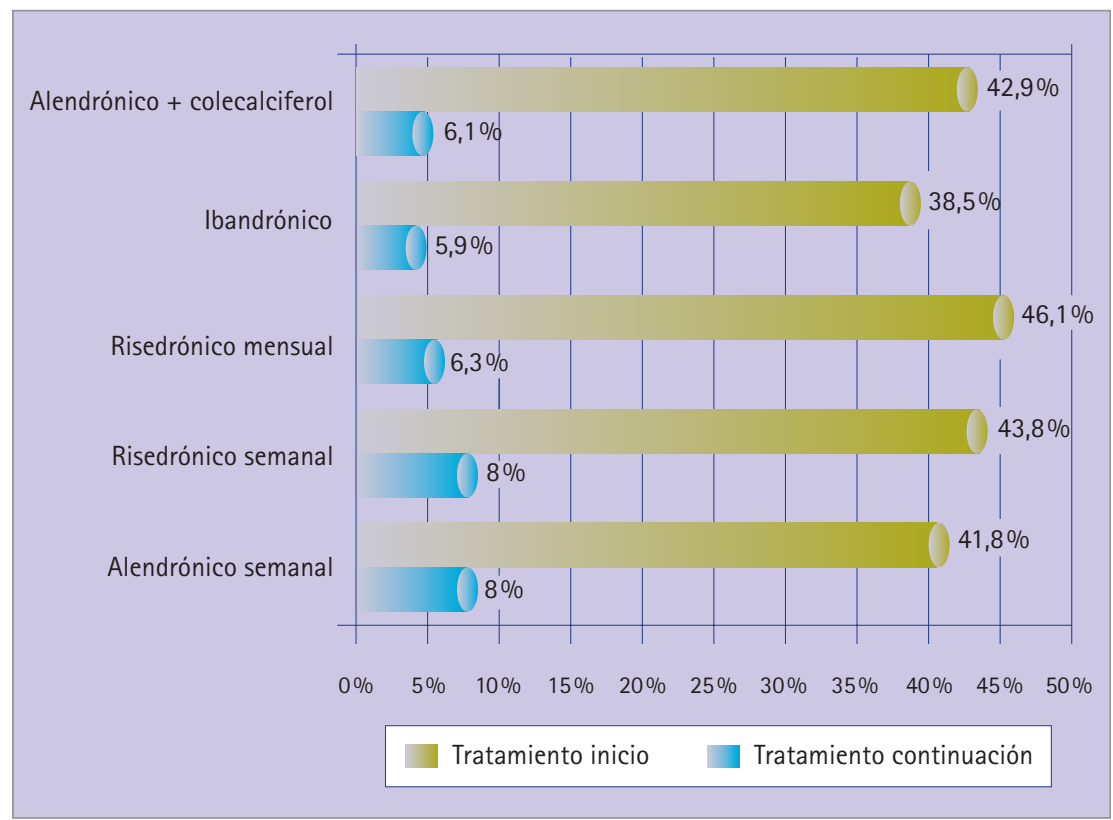

Figura 5 Falta de suplemento de calcio según tipo de tratamiento y principio activo

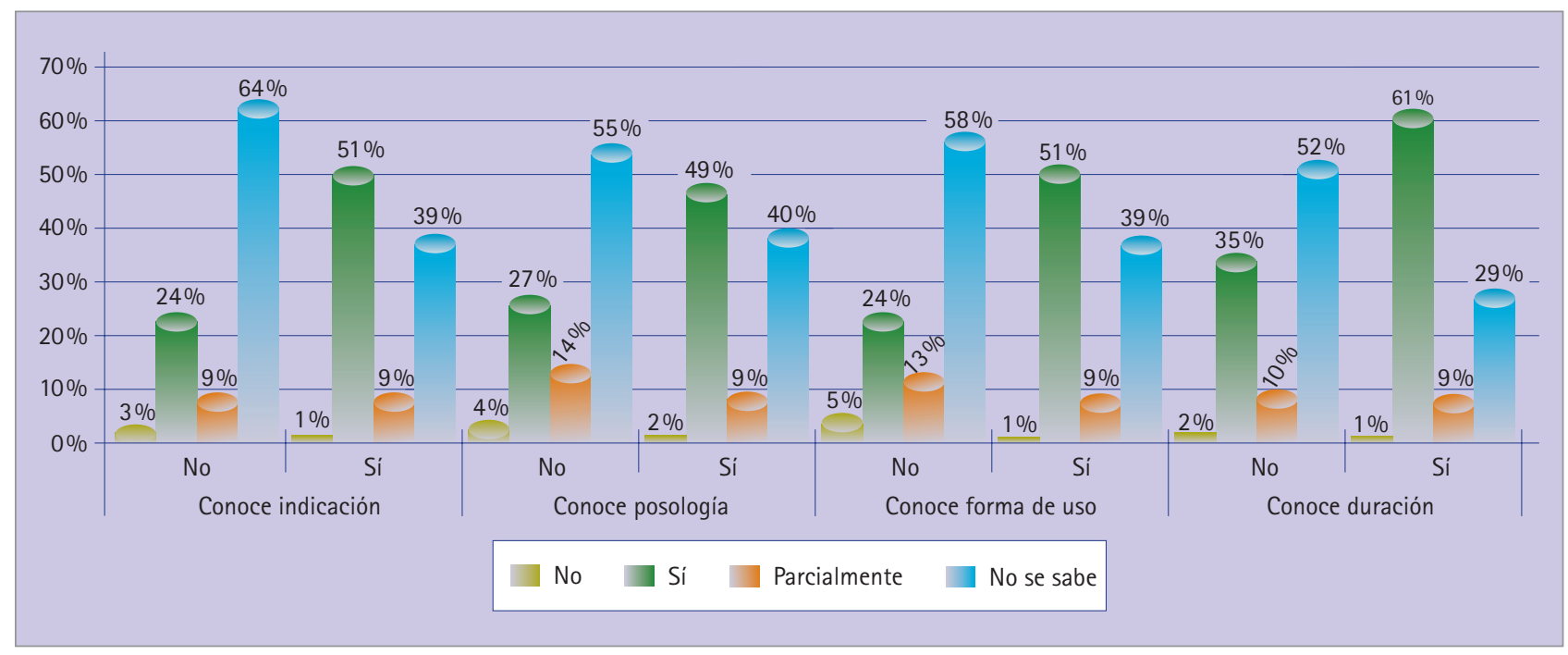

Figura 6 Percepción por el paciente de la efectividad del bifosfonato en relación con la información del proceso de uso

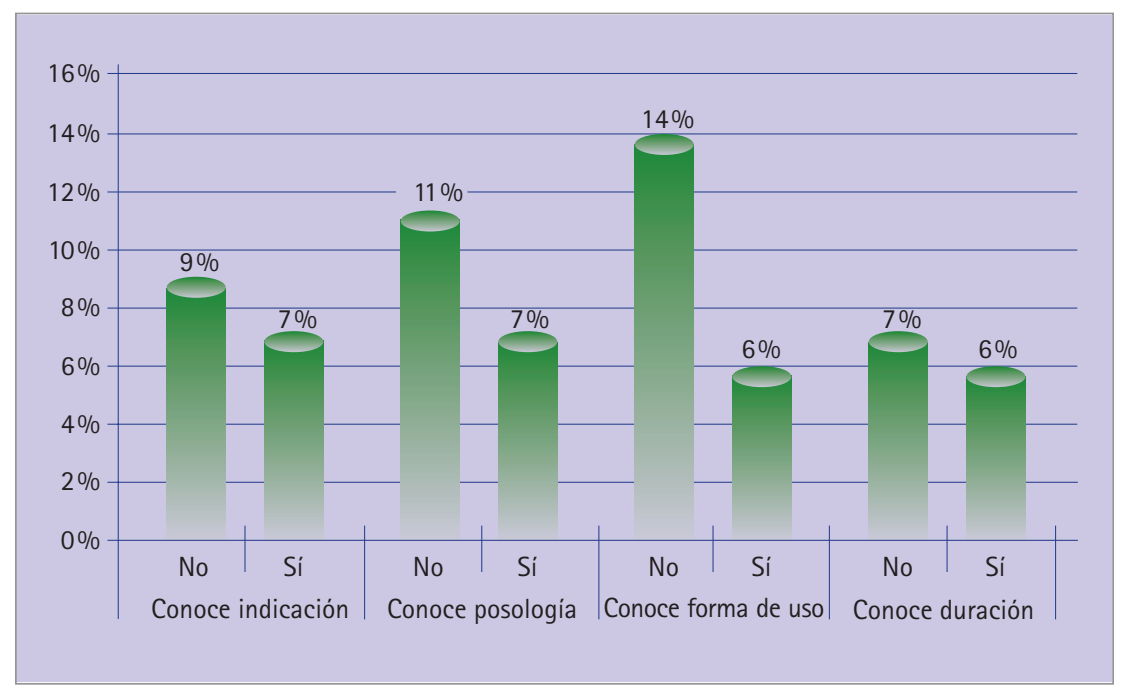

Figura 7 Percepción de inseguridad del paciente en relación con el conocimiento del proceso de uso del bifosfonato
En la figura 5 se muestra la proporción de pacientes que no tomaban suplementos de calcio de los tres principios activos mayoritarios en sus cinco presentaciones más frecuentes y según fuera tratamiento de inicio o de continuación. No se indican los ácidos risedrónico y alendrónico en presentación diaria por ser muy minoritarios.

\section{Percepción de efectividad y seguridad}

En las figuras 6 y 7 se indica la percepción de efectividad y seguridad del paciente según las carencias de información detectadas en los tratamientos de continuación. En los cuatro conceptos que deben conocer los pacientes, cuando se conocen, la percepción de 


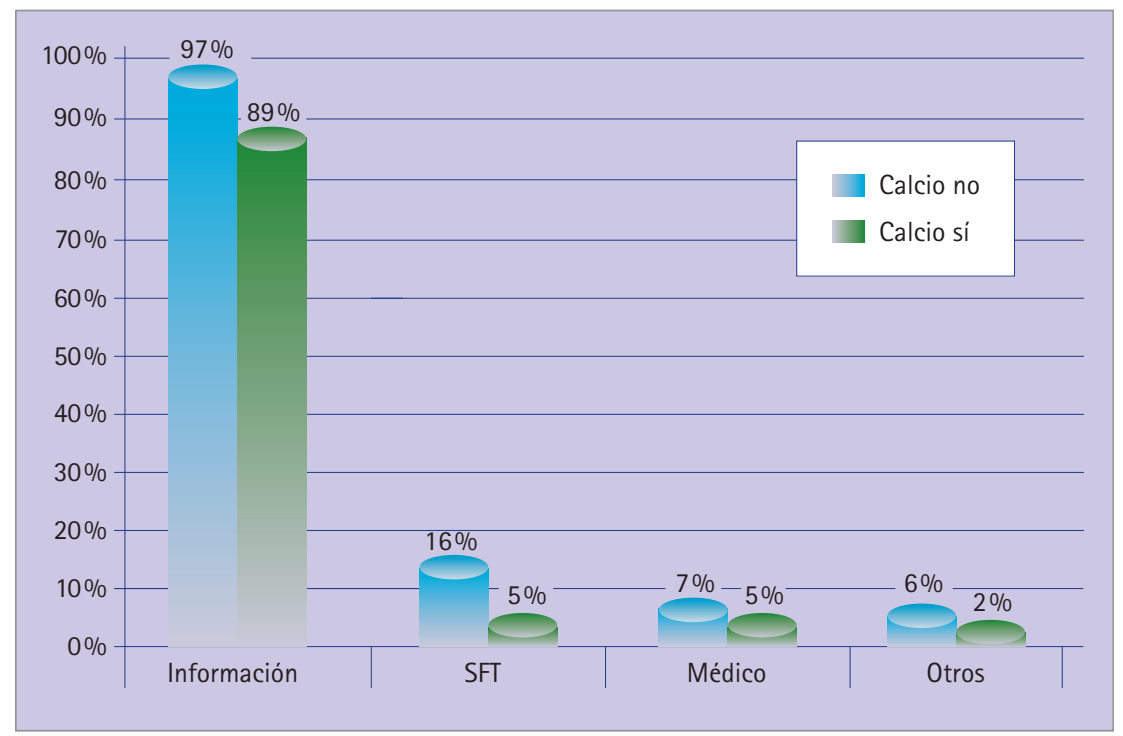

Figura 8 Intervención del farmacéutico en la dispensación, según hubiera o no suplemento de calcio

efectividad y seguridad es mayor. En el caso de efectividad, la proporción de pacientes que dicen no poder apreciar la efectividad "no se sabe" es menor si conocen el medicamento.

\section{Intervención}

Con un mismo paciente se podía intervenir de más de una forma. $\mathrm{Al}$ 90\% de los pacientes se les proporcionó información personalizada del medicamento o educación sanitaria. $\mathrm{Al} \mathrm{6 \%}$ se les ofreció el servicio de seguimiento farmacoterapéutico, al 5\% se derivó al médico y en un 3\% de los casos el farmacéutico intervino de otra forma no tipificada en los registros del estudio.

En la figura 8 se indica la intervención realizada según el paciente tomara calcio o no.

\section{Discusión}

El objetivo principal de D-Valor fue comprobar los conocimientos del paciente sobre su medicación y corregirlos si fuera necesario. En el caso concreto de los bifosfonatos además se hacía especial hincapié en la forma de uso y los suplementos de calcio. Es importante porque las carencias de información en estos aspectos pueden dificultar el uso de los medicamentos y la adherencia al tratamiento. Y esto puede hacerse muy fácilmente en la dispensación.

El resultado del estudio es que los pacientes tienen carencias de información sobre sus medicamentos, que estas carencias afectan negativamen- te a su percepción de efectividad y seguridad de los mismos. El farmacéutico tiene la obligación legal y moral de subsanar estas carencias.

Como era de esperar los pacientes que toman bifosfonatos son predominantemente mujeres de más de 65 años. Los hombres que toman bifosfonatos son muchos menos y suelen ser de mayor edad que las mujeres porque desarrollan la enfermedad más tarde (17).

El principio activo más empleado es ácido alendrónico con o sin colecalciferol, seguido del risedrónico en cualquiera de sus dos presentaciones, pero las diferencias entre los 3 más usados no son muy importantes. Hay una pequeña proporción del 0,8\% de presentaciones diarias, de las que un $0,1 \%$ es de ácido risedrónico $30 \mathrm{mg}$ para uso diario que solo tiene la indicación autorizada de enfermedad de Paget. Aunque el ácido alendrónico también es eficaz en Paget (18), en España no tiene reconocida esa indicación.

Las carencias de información sobre el correcto proceso de uso del bifosfonato son importantes, especialmente en los tratamientos de inicio, ya que solo un $30 \%$ de estos pacientes conoce todo lo que se considera básico, y poco más de la mitad en los tratamientos de continuación, que se supone de los que deberían haber sido informados previamente. La carencia más frecuente es la duración del tratamiento. Se podría pensar que el paciente no ha sido informado por el médico porque todavía no lo tiene decidido. Sin embargo las carencias en indicación, posología y forma de uso no tienen justificación. Un $41 \%$ de los pacientes que inician un tratamiento con bifosfonatos no sabe cómo lo tiene que tomar y esto supone riesgos importantes de efectividad y seguridad (19).

Estas carencias de información se relacionan intensamente con la percepción del paciente. El paciente percibe que el bifosfonato es más efectivo si conoce las características de su tratamiento. El paciente no puede saber la efectividad real del bifosfonato salvo si se hace una densitometría, ya que la osteoporosis no duele ni presenta ningún otro síntoma. Pero no estamos hablando de la efectividad real, sino de la opinión del paciente.

Cuando preguntábamos por efectividad había cuatro posibles respuestas: "no es eficaz", "sí lo es”, "es eficaz parcialmente”, o "no lo sé”. Esta última respuesta, "no se sabe", es la correcta, pero es menos frecuente si el paciente conoce su tratamiento porque entonces predomina la respuesta "sí es eficaz".

Por inseguridad nos referimos a la aparición de los posibles efectos adversos de los bifosfonatos, principalmente los digestivos por ser los más frecuentes, pero no los únicos. A diferencia de la efectividad, la seguridad sí que la puede percibir el paciente, y debería aparecer independientemente de lo que el paciente conozca de su tratamiento. Pero no es así. Si el paciente desconoce su tratamiento la percepción de inseguridad aumenta. Este aumento es más notable si lo que se desconoce es la posología o la forma de uso pasando en este último caso del 6\% al 14\%, más del doble. Esto parece indicar que realmente lo están tomando mal y por ello tienen más problemas.

En las fichas técnicas de todos los bifosfonatos se insiste en la necesidad de asegurar la calcemia. Por otra parte, es algo bastante obvio ya que si pretendemos aumentar la masa ósea habrá que proporcionar el calcio necesario. Esto se puede hacer con la dieta, pero no es frecuente. Menos del 50\% de las mujeres postmenopausicas ingieren un aporte adecuado, y menos del 7\% de las que han sufrido una fractura de cadera (20). Sin embargo, el 12\% no toma suficientes aportes de calcio, llegando al 43\% en los tratamientos de inicio. En los tratamientos de inicio es posible que la no suplementación de calcio sea provisional, o incluso a un error en la respuesta del paciente, pero 
tiene difícil justificación que el 7\% de los tratamientos de continuación no estén suplementados. La falta de calcio es ligeramente superior en los tratamientos semanales con alendrónico y risedrónico. Los pacientes con tratamientos mensuales y los combinados colecalciferol-alendrónico toman un poco más de calcio. La diferencia es pequeña pero estadísticamente significativa.

El farmacéutico intervino más cuando no había suplementos de calcio, facilitaba más información y derivaba más al servicio de seguimiento farmacoterapéutico o al médico. La mayor diferencia entre la intervención con calcio o sin él estaba en la derivación al paciente hacia el servicio de seguimiento farmacoterapéutico, once puntos porcentuales.

No le corresponde al farmacéutico decidir si una persona debe tomar o no bifosfonatos, esa decisión debe tomarla el médico de acuerdo con el paciente. Lo que sí es obligación del farmacéutico es asegurarse de que los pacientes que tomen bifosfonatos conozcan su correcto proceso de uso, para que el del tratamiento consiga el objetivo para el que se ha prescrito, y sea el más efectivo y seguro posible. Se ha demostrado que hay un porcentaje importante de pacientes que no utiliza bien los bifosfonatos porque no tiene la información necesaria; además esa falta de información está relacionada fuertemente con una menor percepción de efectividad y de seguridad.

El farmacéutico está en una posición magnífica para corregir las carencias de información de los pacientes. Solo hace falta mejorar la formación y la implicación del farmacéutico en la dispensación. Pero esa posición mejoraría mucho más si existiera una vía de comunicación segura y ágil con el médico para informarle de las incidencias que se encuentren (21) y para que el médico haga partícipe al farmacéutico de sus objetivos con cada paciente.

\section{Limitaciones del estudio}

El elevado numero de participantes es a la vez una fortaleza y una debilidad. Aunque todos recibieron una formación similar es posible que existiera en algunos casos el sesgo del entrevistador.

Por otra parte, es difícil evaluar con exactitud la información que posee el paciente en el momento de la dispensación. Y que el paciente no tenga la información en ese momento no quiere decir que no sea capaz de obtenerla más tarde.

\section{Agradecimientos}

A los 2.527 farmacéuticos de toda España que entrevistaron a los pacientes en todas las dispensaciones. Ellos fueron los que realmente hicieron el trabajo.

\section{Referencias bibliográficas}

1 Panel de expertos. Foro de Atención Farmacéutica en Farmacia Comunitaria. Guía práctica para los servicios de atención farmacéutica en farmacia comunitaria. Madrid: Consejo General de Colegios Oficiales de Farmacéuticos: 2010. ISBN: 978-84-693-1717-4.

2. Barca Fernández I, Parejo Miguez R, Gutiérrez Martín P, Fernández Alarcón F, Alejandre Lázaro G, López de Castro F. La información al paciente y su participación en la toma de decisiones clínicas. Aten Primaria 2004; 33:361-4. doi:10.1016/S02126567(04)78886-1

3. Baixauli Fernández VJ, Salar Ibáñez L, Barbero González A. Demanda de información en la Farmacia Comunitaria. Pharm Care Esp 2004; 6(3):13644.

4. Márquez Contreras E, Casado Martínez JJ, Márquez Cabeza JJ. Estrategias para mejorar el cumplimiento terapéutico. Form Med Contin Aten Prim. 2001;8:558-73. [Acceso 31/10/2013] Disponible en: http://www.ub.edu/ farmaciaclinica/projectes/webquest/ WQ1/docs/marquez.pdf

5. Sanahuja MA, Villagrasa V, Martínez-Romero F. Adherencia terapéutica. Pharm Care Esp. 2012; 14(4):162-167.

6. Palop Larrea V, Martínez Mir I. Adherencia al tratamiento en el paciente anciano. Inf Ter Sist Nac Salud. 2004;28:113-120.

7. Leal Hernández M, Abellán Alemán J, Casa Pina MT, Martínez Crespo J. Paciente polimedicado: ¿conoce la posología de la medicación?, ¿afirma tomarla correctamente? Aten Primaria. 2004;33(8):451-6. doi:10.1016/ S0212-6567(04)79431-7

8. Centro de Información de medicamentos de Cataluña [Internet]. Cumplimiento terapéutico. [Acceso 31/10/2013] Disponible en: http:// www.cedimcat.info/html/es/dir2455/ doc26897.html

9. Agencia Española del Medicamento [Internet]. Fichas técnicas de medicamentos. [Acceso 30/11/2013]. Dis- ponible en http://www.aemps.gob.es/ cima/fichasTecnicas.do? metodo $=$ detalleForm

10. Pérez Edo L, Alonso Ruiz A, Roig Vilaseca D, García Vadillo A, Guañabens Gay N, Peris P, et al. Actualización 2011 del consenso Sociedad Española de Reumatología de osteoporosis. Reumatol Clin. 2011; 7(6): 357-79. doi:10.1016/j.reuma.2011.05.013

11. National Osteoporosis Foundation 2008 [Internet]. Clinician's guide to prevention and treatment of osteoporosis. [Acceso 30/11/2013]. Disponible en: http://www.seiomm.org/uploads/ documento/d65360173c4bb469e762f9cf18919c6bd405aad1.pdf

12. Zografos GN, Georgiadou D, Thomas D, Kaltsas G, Digalakis M. Drug-induced esophagitis. Dis Esophagus. 2009;22(8):633-7. doi:10.1111/j.14422050.2009.00972.x

13. De Groen PC, Lubbe DF, Hirsch LJ, Daifotis A, Stephenson W, Freedholm D, et al. Esophagitis associated with the use of alendronate. N Engl J Med. 1996 3;335(14):1016-21.

14. Comunicado de la FDA sobre la seguridad de los medicamentos [Internet]. Continúa la revisión sobre la seguridad de los medicamentos para la osteoporosis (bifosfonatos) por vía oral en relación con un probable aumento de riesgo de cáncer de esófago. 21/07/2011. [Acceso 30/11/2013]. Disponible en: http://www.fda.gov/ Drugs/DrugSafety/ucm266340.htm

15. Candelas G, Martínez-Lopez JA, Rosario MP, Carmona L, Loza E. Calcium supplementation and kidney stone risk in osteoporosis: a systematic literature review. Clin Exp Rheumatol. 2012;30(6):954-61.

16. Silver DS. Calciun and vitamin D controversies. Rheum Dis Clin North Am. 2011;37(3):351-63. doi:10.1016/j. rdc.2011.07.005

17. Díaz Curiel M, Moro Álvarez MJ. Epidemiología de la osteoporosis y de las fracturas osteoporóticas. Medicine 2006;9(Extr):15-8.

18. Lojo Oliveira L, Torrijos Eslava A. Tratamiento de la enfermedad ósea de Paget. Reumatol Clin. 2012;8:220-4. doi:10.1016/j.reuma.2011.06.003

19. Strampel W, Emkey R, Civitelli R. Safety considerations with bisphosphonates for the treatment of osteoporosis. Drug Saf. 2007;30(9):755-63. doi:10.2165/00002018-200730090-00003

20. García Vadillo JA. Suplementos de calcio y vitamina D ¿para todos? Reumatol Clin. 2011;7(S2):S34-S39. doi:10.1016/j.reuma.2011.04.008

21. Uribe G, Martínez de la Hidalga G. Médicos y farmacéuticos: éxitos y fracasos de colaboración profesional. Semergen 2002;28(2):86-8. doi:10.1016/ S1138-3593(02)74043-4 